\title{
COVID-19 Pandemic and Endemic Febrile Illnesses: The Dilemma of Exclusion and Diagnosis with Limited Capacities in Aden, Yemen.
}

\author{
Abdulla S. Bin-Gouth \\ Professor of Community Medicine, \\ Hadramout University College of Medicine, Mukalla, Yemen.
}

Yasser A. Baheshem

General Director of the National Malaria Control Program, Aden, Yemen.

Ghanim Y. M. Alsheikh

WHO Collaborating Centre for Public Health Education and Training, Imperial College London, UK.

Former Dean, Hadramout University College of Medicine, Mukalla, Yemen. Corresponding author: alsheikhg@gmail.com

\begin{abstract}
Background: In the beginning of May 2020, Aden was living a state of catastrophic events with the spread of COVID-19 pandemic with accompanying heavy raining season leading to increase of unexpected fever cases. Despite the limited diagnostic capacity, data on causes and trends of febrile illness can be valuable indicators of the causes and trends of fever problems in Aden at the time of COVID-19 pandemic. Objective: to analyze the available data on febrile illness reported in the "CubanYemeni Hospital" in Aden during May-June 2020. Methods: This is a retrospective review of the available data from the hospital registry. Results: Out of 7385 febrile patients, there were 1208 confirmed Malaria cases (16.4\%), 1786 clinically diagnosed Dengue cases (23.3\%), 1855 clinically diagnosed Chikungunya (25\%), 2023 cases of respiratory tract infections-RTI (26.6\%) and only 613 probable COVID-19 cases (8.4\%). The total deaths in this period was 63 , most of them died due to respiratory problems. Conclusions: Malaria is a problem of serious concern in Aden as $16 \%$ of fever in this study were confirmed by laboratory testing while Dengue and Chikungunya were reported frequently but the diagnosis is still based on clinical grounds. As the diagnostic capacity for COVID-19 was limited, the available data underestimated the COVID-19 problem.
\end{abstract}

Key words: COVID-19, Dengue, Chikungunya, Malaria, Fever, Aden, Yemen.

\section{Introduction}

The infectious causes and epidemiology of acute febrile illness (AFI), defined as illness of <1week duration with no identified source, remain poorly characterized in many parts of the world. ${ }^{[1,2]}$ In some regions, such as Sub-Saharan Africa and Southeast Asia, sentinel hospital-based studies have been established to obtain clinical and public health data about the causes of AFI throughout the year and to identify susceptibility patterns and clinical predictors. ${ }^{[2-7]}$

In Yemen, many vector-borne diseases are prevalent, such as Malaria, Dengue and Chikungunya especially after rainy seasons that could bring about local outbreaks. The burden of these diseases in some areas of Yemen like Aden is still uncertain. Aden, in Southern Yemen, was exposed to a heavy rainy season during April, May and June 2020 which coincided with the period of COVID-19 pandemic that led to over expected number of febrile illness cases and deaths. During the period from $1^{\text {st }}$ May to 16 June 2020, there were 2317 deaths in Aden from different causes. ${ }^{[8]}$ The official number of deaths 
from COVID in Aden and the southern governorates till 16 June 2020 was only 244 out of 902 confirmed cases. ${ }^{[9]}$ This underestimated number of cases and deaths raises questions about the other causes of febrile illness and deaths in Aden and what roles, Malaria, Dengue and Chikungunya could play during this situation?

In the beginning of May 2020, Aden was living under catastrophic events comprising the spread of COVID-19 pandemic and the heavy rains that led to unexpected increase in the number of fever cases. This situation stretched the capacity of hospitals to respond; some hospitals were closed. One hospital continued to provide health services and opened a "fever center" of 10 beds in the emergency department to manage the febrile patients. This is the Modern "Cuban-Yemeni Hospital" (CYH). This hospital is a private hospital and located in Al-Mansurah district of Aden providing mainly surgical and trauma care services. The fever center provides first aid, intermediate care within 24 hours admission, referral of the probable COVID patients to the COVID-19 isolation centers. The other febrile patients are treated as outpatients and mostly with diagnosis based on clinical grounds except for Malaria where the confirmation of laboratory test by rapid diagnostic test (RDTs) is entrusted.

Despite the limited diagnostic capacity, data on causes and trends of febrile illness can be valuable indicators of the causes and trends of fever problems in Aden. This study aims at analyzing the available data on febrile illness reported in the "Cuban-Yemeni Hospital" in Aden during May-June 2020 and to present the characteristics and trends of prevailing febrile illness in Aden.

\section{Methods:}

This is a retrospective review of the available data from the hospital registry, the management team of the hospital was also interviewed to clarify associated procedures and data records. The main outcome variable is the causes of fever. The obtained data were entered into the excel program and analyzed using frequency and percentages. Data are presented in tables and graphs.

\section{Results}

A total of 7385 febrile patients out of the 9345 consultations reported in the hospital during the period 13 May-16 June 2020. The proportion of febrile patients attending the hospital is $79 \%$. Out of the 7385 febrile patients, there were 1208 confirmed as Malaria cases (16.4\%), 1786 clinically diagnosed Dengue cases (24,1\%), 1855 clinically diagnosed Chikungunya (25.1\%), 2023 cases of Respiratory Tract Infections (27.4\%) and 613 probable COVID-19 cases $(8.3 \%)$. The total deaths in this period was 63 deaths, most of them died due to respiratory problems (table 1.)

Table 1: Distribution of causes of fever by gender, Aden, May-June 2020.

\begin{tabular}{|lcccc|} 
Causes of Fever & Male & Female & Total & $\begin{array}{c}\text { M : F } \\
\text { Ratio }\end{array}$ \\
\hline Malaria & 677 & 531 & 1208 & $1.27: 1$ \\
\hline Dengue & 913 & 773 & 1686 & $1.18: 1$ \\
\hline Chikungunya & 1148 & 807 & 1855 & $1,42: 1$ \\
\hline COVID-19 & 364 & 250 & 613 & $1.45: 1$ \\
\hline Respiratory Tract Infection (RTI) & 1138 & 885 & 2023 & $1.28: 1$ \\
\hline
\end{tabular}


Figure 1. Causes of febrile illness among 7385 patients attend the emergency department, Cuba-Yemeni hospital in Aden, May-June 2020

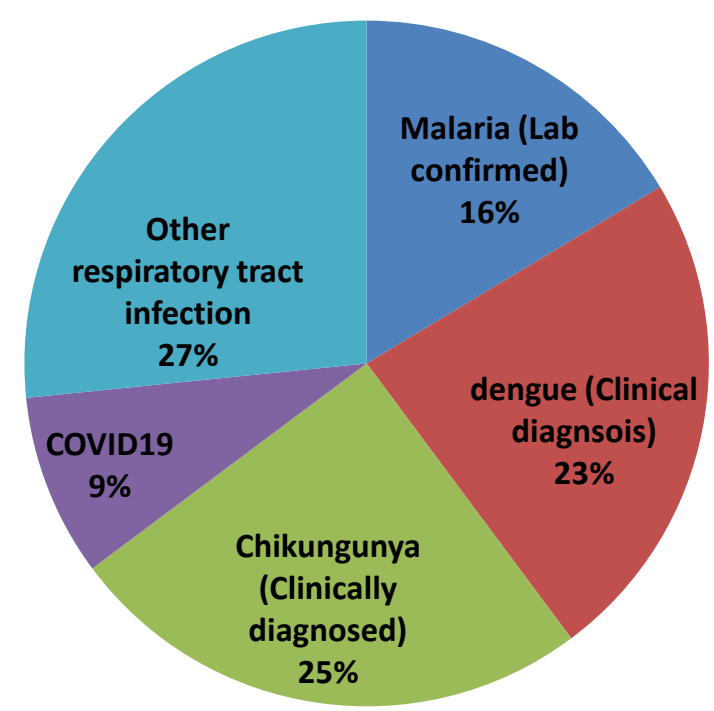

Figure 1. Causes of febrile illness among 7385 patients who attended CYH, May-June 2020.

The results show regular reduction of the fever cases from all causes from Epidemic week 19 to week 23. In general, all fever cases reduced from 1765 in week 19 to 838 cases in week 23 with a reduction of $52.5 \%$ (Figure 2.)

As shown in figure 2, Malaria cases were decreased from 306 in w19 to 148 cases (the reduction was 51.6\%). Dengue were reduced from 444 cases in week 19 to 196 cases in week 23, the reduction was $55.8 \%$ figure 2.) The same findings were observed for Chikungunya where cases reduced from 456 in week 19 to 232 cases in week 23 with a reduction of $49 \%$ (figure 2.)

Due to the limited diagnostic capacity for COVID-19 in Yemen, the study hospital diagnosed COVID19 cases by clinical symptoms and signs plus CT Scan findings of the lungs. Only the high probable COVID-19 cases were referred to the COVID-19 isolation center. A total of 189 probable COVID-19 cases were identified in week 19 which was reduced to 44 cases in week 23 with a reduction rate of $76.7 \%$ (figure 2.) Although this is a good reduction rate when compared with the total cases of RTI which were reduced from 370 cases in week 19 to 258 cases in week 23 , the reduction was only $30.2 \%$ (figure 2.) This low reduction rate raises the question of missing the diagnosis of COVID-19 leading to having a number of probable cases kept underestimated. 


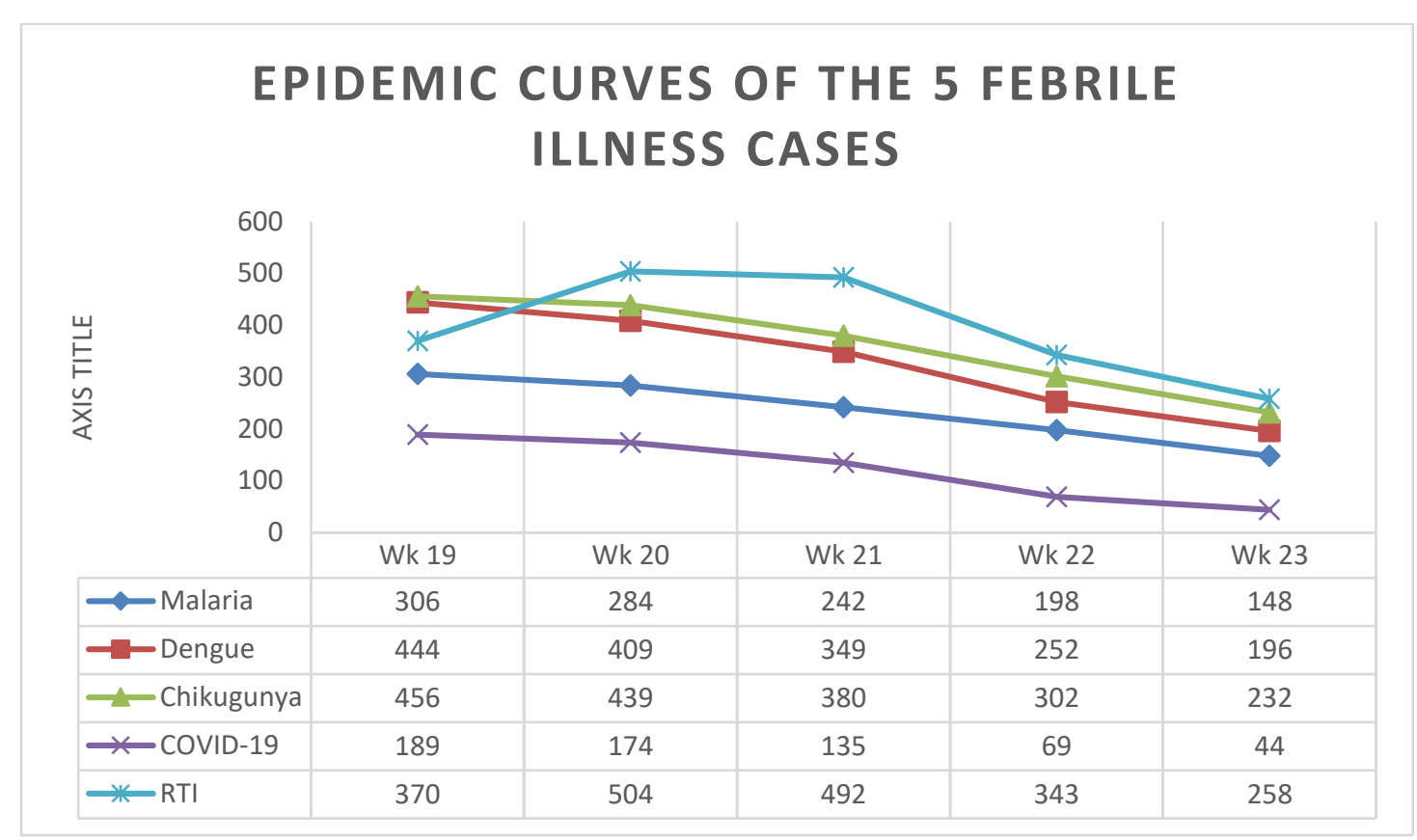

Figure 2. The epidemic curves of the five febrile illness cases, May-June 2020.

\section{Discussion}

Fever is a common presentation at a first point of care in Yemen. In one study in primary health care (PHC) setting in Mukalla, it was found that the respiratory tract infection accounted for $(20.5 \%)$ of common diseases, followed by diarrhea $(12.5 \%)$ and fever $(9 \%) .{ }^{[10]}$

Malaria is one of the most common causes of fever in Yemen. Malaria constituted about $6 \%$ of the total consultations in Yemen after it's rise from 3.9\% in the epidemic week No 22 in 2019 to $6 \%$ in the same week in 2020. ${ }^{[11]}$ In this study showing data from one private hospital, Malaria represented $16 \%$ of the fever cases presented during the same period. In the Zeway Health Center in Ethiopia, Malaria accounted for $17 \%$ of febrile illnesses. ${ }^{[12]}$ In endemic areas of Malaria, the importance of the study of other causes of fever has increased with the decline in the incidence of Malaria as in the case of situation in Asia ${ }^{[13]}$ Other causes of fever other than Malaria include Dengue and Chikungunya fevers. In Latin America, the most frequently reported pathogen was the Dengue virus (DENV) followed by Chikungunya virus. ${ }^{[14]}$

In this study, although Dengue and Chikungunya cases were clinically diagnosed, both ailments can be seriously taken into consideration as $23 \%$ of fever cases were diagnosed as Dengue and $25 \%$ as Chikungunya. Furthermore, the Respiratory Tract Infection (RTI) is found to be the leading cause of morbidity and mortality in Yemen, either in the form of upper respiratory tract infection (URTI) or as lower respiratory tract infection (LRTI). ${ }^{[11]}$ RTI accounted for about $14.2 \%$ of the total consultations in 2019 and increased to $15.7 \%$ during the period between week 19 and week 22 in 2020. The increased percentage in 2020 may be influenced by the spread of COVID-19 in Yemen in May through June 2020, ${ }^{[9,10]}$ In this study, RTI accounted for $26.6 \%$ and probable COVID-19 cases accounted for $8.3 \%$ of all fever cases registered in the study hospital in Aden. In Yemen, till 20 June 2020, there were 919 confirmed cases of COVID-19 out of them 217 cases were from Aden (23.6\%). The total suspected COVID-19 cases (with respiratory symptoms) in Yemen were 2243 cases out of them 293 from Aden (13\%.) ${ }^{[9]}$ In the United Kingdom, general practice consultation rates in England and Wales show that a 
quarter of the population will visit their GPs because of an RTI each year. RTIs are the reason for $60 \%$ of all antibiotic prescribing in general practice. ${ }^{[15]}$

COVID-19 pandemic is heavily spread throughout the world including Yemen with a high anticipation of the worst scenario. The "Médecins Sans Frontières", which runs a COVID-19 centre in Aden, showed that from April 30 to May 17 (18 days) a total of 173 patients were admitted and of whom, at least 68 had died ${ }^{[16]}$ Officials in Aden had reported at least 385 deaths of people with COVID-19-like symptoms from May 7 to May 14, according to Save the Children. That's over 50 deaths a day, a fivefold increase from the 10 deaths a day reported before the $7^{\text {th }}$ May. ${ }^{[17]}$ "COVID-19 is pushing this country even further into the abyss. The surging deaths in Aden suggest that the virus is spreading far faster and further than the number of confirmed cases." [17] Based on the above data, what was identified by the study hospital (CYH) as a probable COVID-19 cases (613 cases during one month) could easily be considered as underestimated.

The trends of all causes of fever show gradual decrease to about 50\% except that for the RTI which was reduced to only $30 \%$. Reduction of fever from all causes at the same period may be explained that Aden was facing a "multi-epidemics" at the same time: COVID-19, Malaria, Dengue and Chikungunya; the situation was complicated by the global spread of COVID-19 and the occurrence of the heavy rains due to climate changes (figure 2).

\section{Conclusions}

In general, all causes of fever including COVID-19 were gradually decreasing from May to June 2020 in Aden. Malaria is a problem of serious concern in Aden. Almost $16 \%$ of fever cases encountered in this study, were confirmed by laboratory tests to be due to Malaria. Although Dengue and Chikungunya were reported frequently but the diagnosis is still based on clinical grounds and not confirmed by laboratory testing. The diagnostic capacity for COVID-19 is limited, making the available data underestimating to the COVID-19 problem. Only highly probable cases of COVID-19 were referred to the isolation center without data about follow-up. High proportion of RTI were reported among adults and most of deaths occurred were due to respiratory problems raising the question of role of COVID-19 among this group of patients.

\section{References}

1. Afifi S, Earhart K, Azab M, Youssef F, Sakka H, Wasfy M, Mansour H, Oun SE, Rakha M, Mahoney F. Hospital-based surveillance for acute illness in Egypt: a focus on community-acquired bloodstream infections. Am J Trop Med Hyg. 2005; 73:392-399.

2. Murdoch D, Woods C, Zimmerman M, Dull P, Belbase R, Keenan A, Scott RM, Archibald LK, Reller LB. The etiology of febrile illness in adults presenting to Patan Hospital in Kathmandu, Nepal. Am J Trop Med Hyg. 2004; 70:670-675.

3. Archibald LK, den Dulk MO, Pallangyo KJ, Reller LB. Fatal Mycobacterium tuberculosis bloodstream infections in febrile hospitalized adults in Dar es Salaam, Tanzania. Clin Infect Dis. 1998; 26:290-296.

4. Ssali FN, Kamya MR, Wabwire-Mangen F, Kasasa S, Joloba M, Williams D, Mugerwa RD, Ellner JJ, Johnson JL. A prospective study of community-acquired bloodstream infections among febrile adults 
admitted to Mulago Hospital in Kampala, Uganda. J Acquir Immune Defic Syndr Hum Retrovirol. 1998; 19:484-4897.

5. Archibald LK, McDonald LC, Rheanpumikankit S, Tansuphaswadikul S, Chaovanich A, Eampokalap B, Banerjee SN, Reller LB, Jarvis WR. Fever and human immunodeficiency virus infection as sentinels for emerging mycobacterial and fungal bloodstream infections in hospitalized patients 15 years old, Bangkok. J Infect Dis. 1999; 180:87-92.

6. Archibald LK, McDonald LC, Nwanyanwu O, Kazembe P, Dobbie H, Tokars J, Reller LB, Jarvis WR. A hospital-based prevalence survey of bloodstream infections in febrile patients in Malawi: implications for diagnosis and therapy. J Infect Dis. 2000; 181:1414-1420.

7. Bell M, Archibald LK, Nwanyanwu O, Dobbie H, Tokars J, Kazembe PN, Reller LB, Jarvis WR. Seasonal variation in the etiology of bloodstream infections in a febrile inpatient population in a developing country. Int J Infect Dis. 2001; 5:63-69.

8. Bin Ghouth AS. Deaths in Aden during the first 17 days in May2020. A technical report to the MOPH\&P, Yemen: 18 May 2020 (in Arabic).

9. MOPH\&P and the Higher Emergency Committee. The daily COVID 19 pandemic report. 17 June 2020-06-18.

10. Ghouth ASB, Break ASB, Bamossa AAB, Baamer ABA, Melkat AA (2019). Common Diseases Presenting in Primary Health Care in Yemen: Investigation of Three Data Sources in PHC Setting. J Community Med Health Res 1(3): 114.

11. Electronic Disease Early Warning System (eDEWS-Yemen) Report. July 2020.Accessed on 10/07/2020: http://moh.gov.ye/

12. Feleke SM, Animut A, Belay M. Prevalence of Malaria among acute febrile patients clinically suspected of having Malaria in the Zeway Health Center, Ethiopia. Jpn J Infect Dis. 2015;68(1):55-59. doi:10.7883/yoken.JJID.2013.062

13. P. Shrestha P, Roberts T, Homsana A, Myat TO, Crump JA, Lubell Y, Newton PN. Febrile illness in Asia: gaps in epidemiology, diagnosis and management for informing health policy. Clin Microbiol Infect. 2018;24(8):815-826. doi:10.1016/j.cmi.2018.03.028.

14. Moreira J, Bressan CS, Brasil P, Siqueira AM. Epidemiology of acute febrile illness in Latin America. Clin Microbiol Infect. 2018;24(8):827-835. doi:10.1016/j.cmi.2018.05.001.

15. Centre for Clinical Practice at NICE (UK). Respiratory Tract Infections - Antibiotic Prescribing: Prescribing of Antibiotics for Self-Limiting Respiratory Tract Infections in Adults and Children in Primary Care. London: National Institute for Health and Clinical Excellence (UK); 2008 Jul. (NICE Clinical Guidelines, No. 69.) Accessed on 09/07/2020:

https://www.ncbi.nlm.nih.gov/books/NBK53632/ 
16. Devi S. Fears of "highly catastrophic" COVID-19 spread in Yemen. The Lancet. 2020;395(10238):1683. doi:10.1016/S0140-6736(20)31235-6.

17. Save the Children. Yemen: deaths due to COVID-like symptoms surge in Aden as hospitals close. Accessed on 11/07/2020: https://www.savethechildren.org.uk/news/media-centre/press-

releases/yemen-deaths-due-to-covid-Aden-hospitals-close 